Hydraulic Engineering Repository

Ein Service der Bundesanstalt für Wasserbau

\title{
Hettler, Achim
}

\section{Die 5. Auflage der Empfehlungen des Arbeitskreises Baugruben}

Verfügbar unter / Available at:

https://hdl.handle.net/20.500.11970/101871

Vorgeschlagene Zitierweise / Suggested citation:

Hettler, Achim (2012): Die 5. Auflage der Empfehlungen des Arbeitskreises Baugruben. In: Bundesanstalt für Wasserbau (Hg.): Neue Normen und Regelwerke in der Geotechnik. Karlsruhe: Bundesanstalt für Wasserbau. S. 49-58. 


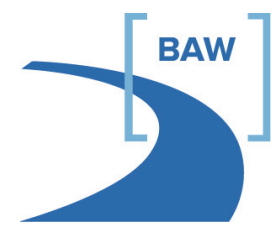

BAW-Kolloquium

Neue Normen und Regelwerke in der Geotechnik am 8. November 2012 im HCC, Hannover

\section{Die 5. Auflage der Empfehlungen des Arbeitskreises Baugruben ${ }^{1}$}

Univ.-Prof. Dr.-Ing. habil. Achim Hettler, Technische Universität Dortmund

\section{Zusammenfassung}

Mit der Einführung des Eurocode 7 einschließlich Nationalem Anhang und ergänzenden Regeln der DIN 1054 wurde eine Anpassung der 4. Auflage der Empfehlungen des Arbeitskreises „Baugruben“ (EAB) der Deutschen Gesellschaft für Geotechnik erforderlich. Im vorliegenden Beitrag werden die wesentlichen Änderungen der 5. Auflage vorgestellt und kommentiert.

\section{Einführung}

Mit der sich abzeichnenden bauaufsichtlichen Einführung der Eurocodes wurde eine Anpassung der 4. Auflage der Empfehlungen an die Vorgaben der DIN EN 1997-1:2009 in Verbindung mit dem Nationalen Anhang DIN 1997-1/NA: 2010-12 und den ergänzenden Regelungen der DIN 1054:2010-12 erforderlich. Alle Empfehlungen wurden gründlich überprüft, soweit erforderlich überarbeitet und an neue Erkenntnisse angepasst. Der erfahrene Anwender wird feststellen, dass die Änderungen in der vorliegenden 5. Auflage verhältnismäßig gering sind. Durchgängig neu sind die Umstellung der Lastfälle auf Bemessungssituationen und die Neuordnung der Grenzzustände der Tragfähigkeit sowie der Gebrauchstauglichkeit. Alle Symbole wurden - soweit sinnvoll und möglich - an die Eurocodes angepasst. Die meisten der seit Jahren bewährten Regelungen konnten jedoch erhalten werden, weil sich die Sicherheitsphilosophie gegenüber der 4. Auflage vom Grundsatz her nicht geändert hat.

Wesentlich überarbeitet wurde dagegen Kapitel 10 „Baugruben im Wasser“. Der Planer muss zukünftig ausführlicher als bisher z. B. auf Risiken aus Erosionsvorgängen, Anisotropie in der Durchlässigkeit und hydraulischem Grundbruch eingehen. Aufgrund der fortgeschrittenen Entwicklung in der Messtechnik und den gestiegenen Anforderungen wurde Kapitel 14 „Messtechnische Überprüfung und Überwachung von Baugrubenkonstruktionen" völlig neu formuliert. Dadurch war eine neue Nummerierung für die einzelnen Empfehlungen erforderlich.

Nachdem keine Einsprüche und Änderungswünsche eingegangen waren, wurde der in der Bautechnik [10] veröffentlichte Entwurf der EB 102 „Anwendung des Bettungsmodulverfahrens“ als Empfehlung unverändert übernommen, siehe auch Brand et al. [3]. Gestrichen wurde dagegen die EB 27, „Anwendung des Traglastverfahrens" wegen der Schwierigkeiten bei der Einführung von Bruchursachen und der Wahl von passenden Lastbildern, wenn sich Fließgelenke beim Verfahren plastisch - plastisch einstellen. Hierzu wird auf die Untersuchungen von Grabe et al. [7] verwiesen sowie auf Vergleichsberechnungen der BAW für den Arbeitskreis, die aber nicht veröffentlicht wurden. Grundsätzlich kann jedoch nach wie vor das Traglastverfahren eingesetzt werden, wenn alle Teilsicherheitsbeiwerte $\gamma_{i}=1,0$ betragen. Ein entsprechender Passus findet sich in der 5. Auflage mit Verweis auf die 4. Auflage. Im Folgenden werden einige der wesentlichen Punkte der Neuerungen ausführlich dargestellt.

\section{Grenzzustände}

Das Handbuch Eurocode 7, Band 1 unterscheidet den Grenzzustand der Tragfähigkeit, der als ULS (UItimate Limit State) bezeichnet wird und den Grenzzustand der Gebrauchstauglichkeit, der mit SLS (Serviceability Limit State) abgekürzt wird.

Beim Grenzzustand ULS werden nach Eurocode 7-1 folgende Fälle definiert:

- EQU: Gleichgewichtsverlust des als starrer Körper angesehenen Tragwerkes ohne Mitwirkung von Bodenwiderständen. Die Bezeichnung ist abgeleitet von "equilibrium“.

\footnotetext{
${ }^{1}$ Mit freundlicher Genehmigung durch den Verlag Ernst \& Sohn und die Chefredaktion wird hier der Beitrag aus der Bautechnik 89 (2012) Heft 9, S. 554 - 599 wiedergegeben.
} 
- STR: Inneres Versagen oder sehr große Verformung des Tragwerkes oder seiner Bauteile, wobei die Festigkeit der Baustoffe für den Widerstand entscheidend ist. Die Bezeichnung ist abgeleitet von "structure“.

- GEO: Versagen oder sehr große Verformung des Baugrundes, wobei die Festigkeit des Bodens oder des Felses für den Widerstand entscheidend ist. Die Bezeichnung ist abgeleitet von „geotechnics“.

- UPL: Gleichgewichtsverlust des Bauwerkes oder Baugrundes infolge von Auftrieb oder Wasserdruck. Die Bezeichnung ist abgeleitet von „uplift“.

- HYD: Hydraulischer Grundbruch, innere Erosion oder Piping im Boden, verursacht durch Strömungsgradienten. Die Bezeichnung ist abgeleitet von „hydraulic“.

Für die Übertragung auf die Vorgaben der DIN 1054 muss der Grenzzustand GEO aufgeteilt werden in GEO-2 und GEO-3:

- GEO-2: Versagen oder sehr große Verformung des Baugrundes im Zusammenhang mit der Ermittlung der Schnittgrößen und der Abmessungen, d. h. bei der Inanspruchnahme der Scherfestigkeit beim Erdwiderstand, beim Gleitwiderstand, beim Grundbruchwiderstand und beim Nachweis der Standsicherheit in der tiefen Gleitfuge.

- GEO-3: Versagen oder sehr große Verformung des Baugrundes im Zusammenhang mit dem Nachweis der Gesamtstandfestigkeit, d. h. bei der Inanspruchnahme der Scherfestigkeit beim Nachweis der Sicherheit gegen Böschungsbruch und Geländebruch sowie, in der Regel, beim Nachweis der Standsicherheit von konstruktiven Böschungssicherungen.

Die bisherigen Grenzzustände der 4. Auflage werden wie folgt ersetzt:

- Dem bisherigen Grenzzustand GZ 1A entsprechen ohne Einschränkung die Grenzzustände EQU, UPL und HYD.

- Dem bisherigen Grenzzustand GZ 1B entspricht ohne Einschränkung der Grenzzustand STR. Hinzu kommt der Grenzzustand GEO-2 im Zusammenhang mit der äußeren Bemessung, d. h. bei Inanspruchnahme der Scherfestigkeit beim Erdwiderstand, beim Gleitwiderstand, beim Grundbruchwiderstand und beim Nachweis der Standsicherheit in der tiefen Gleitfuge.

- Dem bisherigen Grenzzustand GZ 1C entspricht der Grenzzustand GEO-3 im Zusammenhang mit dem Nachweis der Gesamtstandsicherheit, d. h. bei Inanspruchnahme der Scherfestigkeit beim Nachweis der Sicherheit gegen Böschungsbruch und Geländebruch.

Der Nachweis der Standsicherheit von konstruktiven Böschungssicherungen ist in jedem Fall dem Grenzzustand GEO zugeordnet. Je nach konstruktiver Ausbildung und Funktion dürfen sie

- entweder im Sinne des bisherigen Grenzzustandes GZ 1B nach den Regeln des Grenzzustandes GEO-2

- oder im Sinne des bisherigen Grenzzustandes GZ 1C nach den Regeln des Grenzzustandes GEO3 behandelt werden.

\section{Bemessungssituationen}

Die bisherigen Lastfälle werden ersetzt durch Bemessungssituationen. Dabei entsprechen im Grundsatz:

- Lastfall LF 1 der Bemessungssituation BS-P,

- Lastfall LF 2 der Bemessungssituation BS-T,

- Lastfall LF 3 der Bemessungssituation BS-A.

Der Regelfall bei Baugruben entspricht der Bemessungssituation BS-T, der Ausnahmefall der Bemessungssituation BS-A.

Der bisherige Lastfall LF 2/3 wird als Sonderfall zukünftig ersetzt durch die Bemessungssituation BS-T/A. Wie in der 4. Auflage umfasst der Sonderfall neben den Lasten des Regelfalls folgende Einwirkungen: 


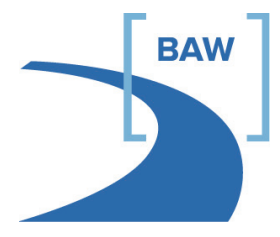

BAW-Kolloquium

Neue Normen und Regelwerke in der Geotechnik am 8. November 2012 im HCC, Hannover

- Fliehkräfte, Bremskräfte und Seitenstoß, z. B. bei Baugruben neben oder unter Eisenbahnen oder Straßenbahnen,

- selten auftretende Lasten und unwahrscheinliche oder selten auftretende Kombinationen von Lastgrößen und Lastangriffspunkten,

- Wasserdruck infolge von Wasserständen, die über den vereinbarten Bemessungswasserstand hinausgehen können, z. B. Wasserstände, bei deren Eintreten die Baugrube überflutet wird oder geflutet werden muss,

- Temperaturwirkungen auf Steifen.

In der Regel ist die Bemessungssituation BS-P - bisher Lastfall LF 1 - für Baugruben nicht maßgebend. Ausnahmen hierzu bilden:

- der Nachweis der Standsicherheit in der tiefen Gleitfuge nach EB 44, bei Baugruben neben Bauwerken,

- der Nachweis der Geländebruchsicherheit nach EB 45, Absatz 7 bei Baugruben neben Bauwerken,

- die Bemessung von Steifen nach EB 52, Absatz 14,

- die Bemessung von Verankerungen für Wände im Vollaushubzustand.

\section{Repräsentative Werte}

Die Kombinationsregeln der Eurocodes können bei Bauwerken zu einer unübersichtlichen Anzahl von Lastfällen führen. Der Arbeitskreis Baugruben war bestrebt, eine möglichst einfache Lösung zu finden. In EB 24, Absatz 7 heißt es:

Zur Ermittlung repräsentativer Werte dürfen bei der Lastermittlung die nachfolgend aufgeführten Kombinationswerte $\Psi$ verwendet werden:

- Bei Baugruben neben Altbauten sollte für die Gründungslasten $\Psi=1,0$ gesetzt werden. Neben Neubauten dürfen die repräsentativen Werte aus der Statik des Tragwerkplaners übernommen werden.

- Werden die Vertikallasten aus Straßen- und Schienenverkehr gemäß EB 55,Abschnitt 2.6 angesetzt, sind Kombinationsbeiwerte $\Psi=1,0$ zu verwenden. Davon abweichend sind auch andere Werte möglich, wenn Vorschriften der jeweiligen Verkehrsbetriebe den Berechnungen zugrunde gelegt werden.

- Allgemein sind bei vereinfachten Lastannahmen nach EB 56, Abschnitt 2.7 für Nutzlasten aus Baustellenverkehr und Baubetrieb sowie nach EB 57, Abschnitt 2.8 für Nutzlasten aus Baggern und Hebezeugen die Kombinationsbeiwerte zu $\Psi=1,0$ zu setzen.

\section{Geotechnische Kategorien}

Neu aufgenommen in die 5. Auflage wurde die EB 106 „Planung und Prüfung von Baugruben“, die als Abschnitt 1.6 dem Kapitel 1 hinzugefügt wurde. In dieser Empfehlung werden Baugruben verschiedenen geotechnischen Kategorien zugeordnet. In Abschnitt 3 heißt es:

„Baugruben sind in eine geotechnische Kategorie GK1, GK2 oder GK3 einzustufen. In Anhang A 5 sind Kriterien in Anlehnung an das Handbuch Eurocode 7, Band 1, Absatz A 2.1.2 für die Einstufung von Baugruben aufgeführt", s. Tabelle 1. 
Tabelle 1: Geotechnische Kategorien für Baugruben gemäß Anhang A 5 der 5. Auflage

\begin{tabular}{|c|c|c|c|}
\hline Situation & $\begin{array}{l}\text { Geotechnische } \\
\text { Kategorie } 1\end{array}$ & $\begin{array}{l}\text { Geotechnische } \\
\text { Kategorie } 2\end{array}$ & $\begin{array}{l}\text { Geotechnische } \\
\text { Kategorie } 3\end{array}$ \\
\hline Baugrund & $\begin{array}{l}\text { mindestens mitteldichte } \\
\text { oder steife Böden } \\
\text { standfester Fels }\end{array}$ & $\begin{array}{l}\text { keine Einordnung in } \\
\text { GK1 oder GK3 }\end{array}$ & $\begin{array}{l}\text { wechselhafte regellose Schich- } \\
\text { ten bindiger und nicht bindiger } \\
\text { Böden } \\
\text { organische Böden } \\
\text { breiige und weiche } \\
\text { bindige Böden } \\
\text { kriechfähige Böden } \\
\text { Fels mit ungünstig verlaufenden } \\
\text { Störzonen oder Trennflächen } \\
\text { Bergsenkungsgebiet, Erdfallge- } \\
\text { biet } \\
\text { heterogene Auffüllungen }\end{array}$ \\
\hline $\begin{array}{l}\text { Grund- } \\
\text { wasser }\end{array}$ & $\begin{array}{l}\geq 0,5 \text { m unter der Bau- } \\
\text { grubensohle }\end{array}$ & $\begin{array}{l}\leq 2 \text { m oberhalb der } \\
\text { Baugrubensohle } \\
\text { kann mit üblichen Maß- } \\
\text { nahmen abgesenkt } \\
\text { werden } \\
\text { wird mit Spundwänden } \\
\text { und unverankerten } \\
\text { Sohlen gehalten }\end{array}$ & $\begin{array}{l}>2 \text { m oberhalb der Baugruben- } \\
\text { sohle } \\
\text { Umströmung der Baugruben- } \\
\text { wände } \\
\text { Horizontale/vertikale Durchläs- } \\
\text { sigkeit > } 3 \\
\text { Setzungsweiche Böden im Ein- } \\
\text { flussbereich der Grundwasser- } \\
\text { absenkung }\end{array}$ \\
\hline $\begin{array}{l}\text { Baugruben- } \\
\text { wand }\end{array}$ & $\begin{array}{l}\text { unverankerte oder ge- } \\
\text { stützte Spundwände } \\
\text { und Trägerbohlwände } \\
\text { bis } 3 \text { m Baugrubentiefe } \\
\text { Normverbau nach } \\
\text { DIN } 4124 \\
\text { Böschungen bis } 3 \mathrm{~m} \\
\text { Unterfangung nach } \\
\text { DIN } 4123 \text { mit einer } \\
\text { freien Höhe } \leq 0,5 \mathrm{~m}\end{array}$ & $\begin{array}{l}\text { Baugrubenwände bis } \\
10 \text { m Baugrubentiefe } \\
\text { Baugrubenwände als } \\
\text { Bohrpfahl- und Schlitz- } \\
\text { wände } \\
\text { Unterfangung nach } \\
\text { DIN } 4123 \text { mit einer } \\
\text { freien Höhe }>0,5 \mathrm{~m}\end{array}$ & $\begin{array}{l}\text { Baugruben neben verschie- } \\
\text { bungs- und setzungsempfindli- } \\
\text { chen Gebäuden } \\
\text { Baugrubenwände, die geringe } \\
\text { Verschiebungen aufweisen müs- } \\
\text { sen } \\
\text { Baugrubenwände mit mehr als } 2 \\
\text { Steifen- oder Ankerlagen } \\
\text { Baugrubenwand als Stützkon- } \\
\text { struktion durch eine Bodenver- } \\
\text { festigung } \\
\text { umströmte Baugrubenwände }\end{array}$ \\
\hline Dichtsohle & keine Dichtsohle & $\begin{array}{l}\text { unverankerte Unter- } \\
\text { wasserbetonsohlen } \\
\text { annähernd undurchläs- } \\
\text { siger Boden }\end{array}$ & $\begin{array}{l}\text { verankerte Unterwasserbeton- } \\
\text { und Bodenverfestigungssohlen } \\
\text { tiefliegende Dichtsohlen }\end{array}$ \\
\hline $\begin{array}{l}\text { Verankerun- } \\
\text { gen der } \\
\text { Baugruben- } \\
\text { wände }\end{array}$ & $\begin{array}{l}\text { keine Verankerung der } \\
\text { Baugrubenwände }\end{array}$ & $\begin{array}{l}\text { maximal eine Ver- } \\
\text { pressankerlage }\end{array}$ & $\begin{array}{l}\text { Lage des Verankerungskopfes } \\
\text { unterhalb des Grundwasserspie- } \\
\text { gels } \\
\text { Verankerungen mit Nägeln ge- } \\
\text { mäß DIN EN } 14490 \text { oder Mikro- } \\
\text { pfählen } \\
\text { Verankerung in Unterfangungs- } \\
\text { körpern }\end{array}$ \\
\hline $\begin{array}{l}\text { Verankerung } \\
\text { der Sohle }\end{array}$ & $\begin{array}{l}\text { keine Verankerung der } \\
\text { Sohle }\end{array}$ & $\begin{array}{l}\text { keine Verankerung der } \\
\text { Sohle }\end{array}$ & $\begin{array}{l}\text { Alle Arten von Sohlverankerun- } \\
\text { gen }\end{array}$ \\
\hline
\end{tabular}




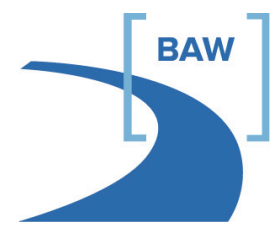

BAW-Kolloquium

Neue Normen und Regelwerke in der Geotechnik am 8. November 2012 im HCC, Hannover

\section{Erddruckfragen}

\subsection{Mindesterddruck}

Der Ansatz eines Mindesterddrucks bei Böden mit Reibung und Kohäsion leitete sich aus zwei Überlegungen ab:

- Einerseits erschien es ganz allgemein nicht hinnehmbar, wenn sich aufgrund des negativen Erddruckanteils infolge von Kohäsion ein sehr geringer Erddruck ergab, für den rechnerisch eine Sparversion eines Verbaus ausreichte, z. B. eine einmal gestützte Trägerbohlwand aus Bohlträgern IPB 100 bei 8,00 m Baugrubentiefe, oder im Grenzfall gar kein Erddruck, so dass sich ein Verbau zumindest rechnerisch erübrigte.

- Andererseits bestand die Gefahr, dass bei stark bindigen Böden die zu erwartende Wandbewegung in der Größenordnung von 1 \%o der Wandhöhe nicht ausreichte, um den Erdruhedruck auf den aktiven Erddruck absinken zu lassen.

Der gewählte Ansatz für Größe und Verteilung des Mindesterddrucks stützt sich auf Plausibilitätsüberlegungen und lässt sich nicht rein theoretisch begründen.

Die Idee eines Mindesterddrucks geht auf Weißenbach zurück. Gestützt auf Messungen an Baugruben, wurde 1970 der erste Entwurf einer Empfehlung zum Mindesterddruck in EB 4, Absatz 3 veröffentlicht [15]. Die Grundidee ist seitdem unverändert geblieben. Der Erddruck aus Bodeneigengewicht und Kohäsion ist zunächst auf der Grundlage der klassischen Erddrucktheorie mit den Scherparametern $\varphi$ und c zu ermitteln. Dessen Resultierende ist mit der Erddrucklast aus Mindesterddruck zu vergleichen. Für die weitere Berechnung ist dann der ungünstigere Ansatz zugrunde zu legen. Erddrücke aus Auflasten waren stets zusätzlich anzusetzen [17]. Im Laufe der Jahre kam es zu Veränderungen im Detail, z. B. wird heute der Mindesterddruck nicht mehr wie anfangs mit einem Beiwert von $\mathrm{K}=0,20$, sondern auf der Grundlage eines Ersatzreibungswinkels $\varphi_{\text {Ers }}=40^{\circ}$ berechnet. In der Fassung der Empfehlungen von 1972 [16] durfte zwar die Erddrucklast aus Auflasten um einen Kohäsionsanteil vermindert werden. Dieser Anteil musste dann jedoch wieder dem Anteil aus Bodeneigengewicht zugeschlagen werden, vgl. Bild EB 7-1 in [16].

Einen anderen Weg beschreitet die DIN 4085-2007-1, neueste, korrigierte Ausgabe vom Mai 2011. Die Regelungen der DIN 4085 können zwar im Sinne der EAB verstanden werden, es ist jedoch auch eine andere Auslegung möglich. Demnach wird die Erddruckresultierende - oder auch als Variante die Erddruckordinate - aus Bodeneigengewicht, Kohäsion und Auflasten mit dem Mindesterddruck verglichen. Dies führt zu einer Verringerung der Einwirkungen auf Stützwände und damit zu einem reduzierten Sicherheitsniveau. Eine Begründung dafür fehlt.

Der Arbeitskreis „Baugruben“ hat diese Frage ausführlich diskutiert und in zwei Sitzungen eingehend behandelt. Wie hoch das Sicherheitsrisiko einzuschätzen ist, ist schwer überschaubar, zumal bei Tonböden auch der Porenwasserdruck eine Rolle spielen kann. Es wurde beschlossen, den seit mehreren Jahrzehnten bewährten Vorschlag von Weißenbach beizubehalten. Zur Vermeidung von Missverständnissen werden in der 5. Auflage wie in der Vergangenheit konsequent die Erddruckkräfte und Erddruckverteilungen aus Auflasten mit dem charakteristischen Reibungswinkel ermittelt. Der Arbeitskreis „Baugruben" hat aber auch akzeptiert, dass bei einem hohen Kohäsionsanteil in besonderen Fällen der Erddruck aus Auflasten teilweise durch rechnerische Zugspannungen reduziert werden darf. Obwohl Untersuchungen zur Auswirkung hoher Kohäsionswerte bei Auflasten fehlen, wurde eine Öffnungsklausel eingeführt. In EB 6, Absatz 4 der 5. Auflage wird zu Nutzlasten für den Fall, dass bei Bodeneigengewicht und Kohäsion der Mindesterdduck maßgebend ist, folgende Regelung vorgeschlagen:

„Auch wenn für bindige Schichten die Größe der Erddrucklast aus Bodeneigengewicht und Kohäsion entsprechend EB 4, Absatz 3b) (Abschnitt 3.2) mit Hilfe eines Ersatzreibungswinkels berechnet wird, ist der Erddruck aus großflächigen lotrechten Gleichlasten einschließlich der Lasten bis $p_{k}=10 \mathrm{kN} / \mathrm{m}^{2}$, sowie aus Linien- und Streifenlasten stets entsprechend Absatz 3a) mit dem charakteristischen Reibungswinkel $\varphi^{\prime}{ }_{k} z u$ ermitteln. In der Regel gilt dies auch für Lasten $p_{k}>10 \mathrm{kN} / \mathrm{m}^{2}$. In Ausnahmefällen dürfen bei einer Kohäsion $\mathrm{c}^{\prime}{ }_{\mathrm{k}}>30 \mathrm{kN} / \mathrm{m}^{2}$ die so ermittelten Erddrücke aus Auflasten mit rechnerischen Zugspannungen 
aus Bodeneigengewicht und Kohäsion angemessen verrechnet werden, sofern genauere Untersuchungen durchgeführt werden und ausreichende örtliche Erfahrungen vorliegen."

\subsection{Erddruckneigungswinkel bei Schlitzwänden}

Seit vielen Jahren darf der Wandreibungswinkel bei Schlitzwänden, deren Oberfläche als weniger rau eingestuft wird, betragsmäßig nicht höher als $\left|\delta_{p}\right| \leq 1 / 2 \varphi$ angesetzt werden. Auf der anderen Seite zeigen Erfahrungen von Firmen bei der Herstellung suspensionsgestützter Pfähle, dass auch höhere Wandreibungswinkel möglich sind. Mittlerweile liegen auch neuere Forschungsarbeiten von Arwanitaki [2] sowie von Arwanitaki, König und Triantafyllidis [1] zu Schlitzwänden vor, die ebenfalls eine höhere Wandreibung belegen. Allerdings liegen noch nicht für alle Bodenarten Untersuchungen vor, so dass noch Forschungsbedarf besteht, vgl. Triantafyllidis in [1].

Um den neuen Erkenntnisstand zu berücksichtigen, wurde EB 89, Absatz 3c in der 5. Auflage entsprechend erweitert:

„Als „weniger rau“ darf die Oberfläche einer Schlitzwand eingestuft werden, sofern die Filterkuchenbildung gering ist, z. B. bei Schlitzwänden in bindigem Boden. Erfahrungsgemäß gilt dies auch bei Schlitzwänden in nichtbindigem Boden. Sofern aber bei der Schlitzwandherstellung durch geeignete Maßnahmen die Ausbildung eines Filterkuchens vermieden werden kann oder eine stark unebene Wandoberfläche erreicht wird, darf auch ein betragsmäßig höherer Erddruckneigungswinkel als $|\delta p|=1 / 2 \varphi$ angesetzt werden."

\section{$7 \quad$ Bemessung der Einzelteile}

\subsection{Vertikale Tragfähigkeit bei Spundwänden}

In Abstimmung mit dem Arbeitsausschuss „Ufereinfassungen“ wird zukünftig der Fußwiderstand bei Spundwänden nicht mehr mit dem Verfahren von Radomski [13], sondern aus dem Sondierspitzendruck und der Stahlfläche ermittelt.

Die Fußfläche sowie die Mantelfläche bei Spundwänden darf nach EB 85, Absatz 3b und Bild EB 85-1 der 5. Auflage ermittelt werden. Dort heißt es:

„Für die Ermittlung der charakteristischen Fußwiderstände nach EB 84, Absatz 2 c) (Abschnitt 4.8) gilt Folgendes: Bei Spundwänden ergibt sich die maßgebende Fuß- bzw. Aufstandsfläche aus der vorhandenen Stahlquerschnittsfläche nach Bild EB 85-1 a)."

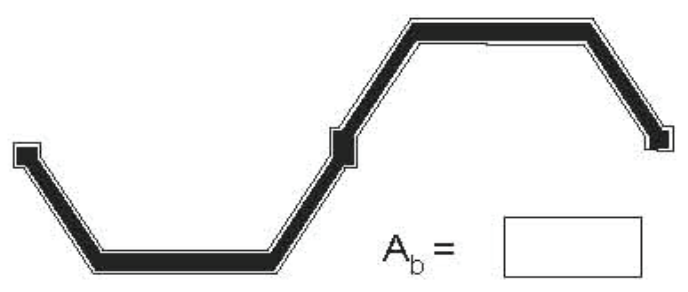

a) Aufstandsfläche

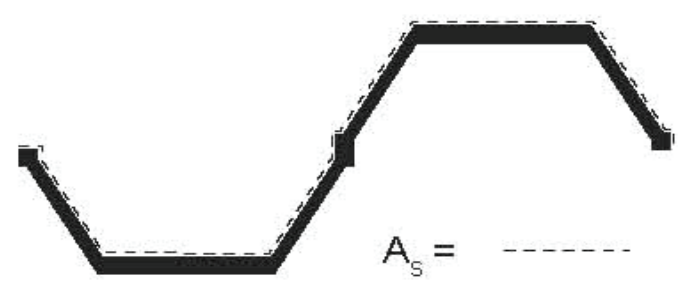

b) Mantelfläche

Bild EB 85-1. a) Wirksame Aufstandsfläche und b) Mantelfläche bei gerammten Wellenspundwänden

Angaben zu charakteristischen Werten für Spitzendruck $q_{b, k}$ und Mantelreibung finden sich im Anhang A10 der 5. Auflage. 


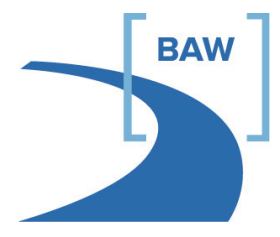

BAW-Kolloquium

Neue Normen und Regelwerke in der Geotechnik am 8. November 2012 im HCC, Hannover

\subsection{Lastfall Ausfall eines Verpressankers}

Der Lastfall „Ausfall eines Verpressankers“ ist immer wieder Gegenstand von Anfragen und Diskussionen. In EB 86, Absatz 5, der 5. Auflage wird folgende Regelung vorgeschlagen:

Auf konstruktive Maßnahmen oder rechnerische Nachweise im Hinblick auf den möglichen Ausfall eines Verpressankers darf verzichtet werden, wenn die folgenden Bedingungen erfüllt sind:

a) Jeder Anker (Kurzzeitanker) wird bei der Abnahmeprüfung mit $P_{p}=1,5 \cdot P_{k}$ geprüft. Es ist hierfür der Nachweis der inneren Tragfähigkeit des Zuggliedes für den Spannvorgang zu führen.

b) Es kommen Litzen- oder Einstabanker zum Einsatz. Bei Litzenankern ist nachzuweisen, dass bei Ausfall einer Litze die restlichen Litzen in der Lage sind, die Ankerkraft $P_{d} z u$ übernehmen, wobei als Bemessungssituation BS-A angesetzt werden darf. Dies kann bei vier oder mehr Litzen als erfüllt angesehen werden.

c) Die tragenden Teile des Ankerkopfes sind hinter der Vorderkante der Bohlträger, der Spundwand, der Pfahl- oder Schlitzwand möglichst weitgehend zu versenken, wenn Gefährdungen durch den Baubetrieb nicht anderweitig ausgeschlossen werden können.

Der Absatz 5 d) der 4. Auflage wurde gestrichen. Grundsätzlich ist jedoch bei Litzenankern auf eine ausreichende Vorspannung zu achten, um eine sichere Verkeilung zu gewährleisten.

Hinweis: Im Beitrag zur Bautechnik wurde irrtümlicherweise der Text der 4. Auflage übernommen.

\section{Baugruben im Wasser}

Kapitel 10 „Baugruben im Wasser“ wurde wesentlich überarbeitet. Der Begriff Strömungsdruck wurde durchgängig durch den Begriff Strömungskraft ersetzt, der aus mechanischer Sicht zutreffender ist und den vektoriellen Charakter der Einwirkung besser kennzeichnet.

Werden zur Absenkung des Grundwassers Brunnen innerhalb einer Baugrube angeordnet, kommt es zu einer Umströmung des Wandfußes und in der Regel zu Richtungsänderungen der Gradienten und damit der Strömungskräfte. In diesen Fällen müssen ausführlicher als bisher Risiken aus Erosionsvorgängen untersucht werden, z. B. Kontakterosion an Schichtgrenzen oder die innere Filterstabilität der Schichten, s. [4]. In Zweifelsfällen sind die Auswirkungen einer Anisotropie näher zu verfolgen, wenn die horizontale Durchlässigkeit wesentlich größer ist als die vertikale, s. EB 59, Absatz 4b der 5. Auflage. Infolge einer Anisotropie konzentriert sich der Potentialabbau im Vergleich zu isotropen Bedingungen auf der Baugrubenseite zwischen Wandfuß und Baugrubensohle, vgl. Mittag und Richter [11] sowie Ziegler und Aulbach [19]. Dadurch erhöht sich z. B. die Gefahr eines hydraulischen Grundbruchs verbunden mit einer Quellbildung oder rückschreitenden Erosion. Der Nachweis einer Anisotropie dürfte in praktischen Fällen schwierig sein. Aufgrund von Streuungen in der Kornverteilung und Feinschichtung wird es kaum gelingen, z. B. durch Laborversuche an Proben mit unterschiedlicher Durchströmungsrichtung einen Anisotropiefaktor zu ermitteln. In der Regel wird man auf numerische Simulation und Parameterstudien angewiesen sein, eventuell in Verbindung mit einer messtechnischen Überwachung während der Bauausführung. Reagiert das System empfindlich auf unterschiedliche Durchlässigkeitsverhältnisse, wird man im Einzelfall die Konstruktion entsprechend sicherer ausführen müssen, z. B. durch Verlängerung der Einbindetiefen.

Dreidimensionale Strömungsverhältnisse, z. B. in den Baugrubenecken, können ebenfalls zu weitaus größeren Strömungskräften führen als im zweidimensionalen isotropen Fall. Diese Erkenntnisse sind nicht neu, wie z. B. die Ausführungen von Weißenbach 1981 im Grundbautaschenbuch zeigen [18], der sich wiederum auf Untersuchungen von Davidenkoff und Franke in den 1960er Jahren bezieht [5, 6]. In der Fachwelt scheint man jedoch erst durch Schadensfälle und Schwierigkeiten bei der Ausführung wieder stärker die Aufmerksamkeit auf diese Themen zu richten. Durch die großen Fortschritte in der EDV stehen seit einigen Jahren der Praxis entsprechende Programme für eine Modellierung von dreidimensionalen Strömungsvorgängen oder anisotropen Verhältnissen zur Verfügung. Eine Übersicht über die Literatur zu hydraulischem Grundbruch, auch zum Nachweis bei Auflastfiltern, offene Fragen und neue Erkenntnisse geben Hettler [8] sowie Odenwald und Herten [12]. 
Die Empfehlung EB 62 „Nachweis der Sicherheit gegen Aufschwimmen“ wurde insbesondere beim Nachweis von rückverankerten Betonsohlen neu formuliert. Anlass waren Diskussionen im Arbeitskreis „Pfähle“ der Deutschen Gesellschaft für Geotechnik insbesondere zur Frage der Einleitung von seitlichen Kräften in die Betonsohle und deren Verteilung, s. Hettler und Morgen [9].

\section{Weitere Hinweise}

Der Arbeitskreis „Baugruben“ dankt allen, die in der Vergangenheit durch Zuschriften oder auf andere Weise die Ausschussarbeit gefördert haben, und bittet auch für die Zukunft um diese Unterstützung.

Aktuell setzt sich der Arbeitskreis aus folgenden Mitgliedern zusammen:

Univ.-Prof. Dr.-Ing. habil. A. Hettler, Dortmund (Obmann)

Dipl.-Ing. U. Barth, Mannheim

Prof. Dr.-Ing. K.-M. Borchert, Berlin

Dipl.-Ing. Th. Brand, Berlin

Dipl.-Ing. P. Gollub, Essen

Dipl.-Ing. W. Hackenbroch, Duisburg

Dipl.-Ing. R. Haussmann (Schrobenhausen)

Dr.-Ing. M. Herten, Karlsruhe

Dipl.-Ing. H.-U. Kalle, Hagen

Univ.-Prof. (em.) Dr.-Ing. H. G. Kempfert, Hamburg

Dr.-Ing. St. Kinzler, Hamburg

Dr.-Ing. F. Könemann, Dortmund

Univ.-Prof. Dr.-Ing. habil. Ch. Moormann, Stuttgart

Dipl.-Ing. Ch. Sänger, Stuttgart

Dipl.-Ing. W. Vogel, München

Gäste: Dipl.-Ing. Dr. Robert Hofmann,

Univ.-Prof. Dr.-Ing. habil. Eugen Perau

\section{Literaturhinweis}

[1] Arwanitaki, A., König, D., Triantafillydis, T.: Zum Kontaktverhalten zwischen suspensionsgestützten Ortbetonwänden und dem anstehenden Boden. Bautechnik 84 (2007), Heft 11, S. 781-792.

[ 2] Arwanitaki, A.: Über das Kontaktverhalten einer Zweiphasen Schlitzwand und nichtbindigen Böden. Schriftenreihe des Lehrstuhls für Grundbau, Boden und Felsmechanik, Ruhr-Universität Bochum, Heft 41, 2009.

[ 3] Brand, T., Bastian, D.; Hillmann, S.: Die Berechnung von Baugruben mit dem Bettungsmodulverfahren nach EB 102. Bautechnik 88 (2011), Heft 10, S. 694-706.

[ 4] Busch, K.-F., Luckner, L.,Timer, K.: Geohydraulik, 3. Neubearbeitete Auflage, Borntraeger, 1993

[5] Davidenkoff, R. und Franke, L.: Untersuchung der räumlichen Sickerströmung in eine umspundete Baugrube in offenen Gewässern. Die Bautechnik 42 (1965), H. 9, S. 298.

[6] Davidenkoff, R. und Franke, L.: Räumliche Sickerströmung in eine umspundete Baugrube im Grundwasser. Die Bautechnik 43 (1966), H. 12, S. 401.

[ 7] Grabe, J., Schümann, B., Katzmann, A.: Anwendung der Fließgelenktheorie auf Baugruben, Bautechnik 85 (2008), S. 443-453.

[ 8] Hettler, A.; Hydraulischer Grundbruch: Literaturübersicht und offene Fragen, Bautechnik 85(2008),H. 9, S. 578-584.

[ 9] Hettler, A., Morgen, K.; Nachweis der Sicherheit gegen Aufschwimmen bei Baugruben mit verankerten Betonsohlen, Bautechnik 85 (2008), H. 6, S. 374-380.

[10] Hettler, A.: Empfehlung EB 102 des Arbeitskreises „Baugruben“ der DGGT zur Anwendung des Bettungsmodulverfahrens. Bautechnik 88 (2011), Heft 9, S. 640-645.

[11] Mittag, J., Richter, Th.: Grundwasserabsenkungen und Grundwasserentspannungen/Risiken und wirtschaftliche Chancen, Veröffentl. Grundbauinstitut der TU Berlin, H. 47, 2009, S. 65-76.

[12] Odenwald, B., Herten, M.: Hydraulischer Grundbruch: neue Erkenntnisse, Bautechnik 85 (2008), H. 9,S. 585-595. 


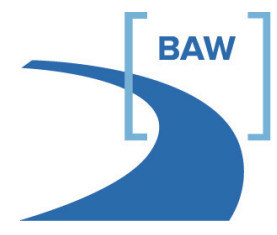

BAW-Kolloquium Neue Normen und Regelwerke in der Geotechnik am 8. November 2012 im HCC, Hannover

[13] Radomski, H.: Untersuchungen über den Einfluß der Querschnittsform wellenförmiger Spundwände auf die statischen und rammtechnischen Eigenschaften. Mitteilungen des Instituts für Wasserwirtschaft, Grundbau und Wasserbau der Universität Stuttgart, Heft 10 (1968).

[14] Saucke, U.: Nachweis der Sicherheit gegen Erosion für körnige Erdstoffe, Geotechnik 29 (2006), H. 1, S. 43-54.

[15] Weißenbach, A.: Empfehlungen des Arbeitskreises „Baugruben“ der Deutschen Gesellschaft für Erdund Grundbau, Bautechnik 47 (1970), H. 7, S. 223-233.

[16] Weißenbach, A.: Empfehlungen des Arbeitskreises „Baugruben“ der Deutschen Gesellschaft für Erdund Grundbau, Bautechnik 49 (1972), H. 6, S. 192-204, H. 7, S. 225-239,

[17] Weißenbach, A.: Baugruben, Teil II: Berechnungsgrundlagen. Berlin/München/Düsseldorf: W. Ernst \& Sohn 1975.

[18] Weißenbach, A.: Baugrubensicherung. Grundbau-Taschenbuch, 3. Auflage Teil 2, S. 887-982. Berlin: Ernst \& Sohn 1992.

[19] Ziegler, M., Aulbach, B.: Zur Sicherheit gegen hydraulischen Grundbruch, Vorträge 31. Baugrundtagung, Deutsche Gesellschaft für Geotechnik, 2010 
